\title{
PCR as a Rapid Diagnostic Tool for Detection of Dermatophytes
}

\author{
Kalyani Putty $^{2}$, J. Shiva Jyothi ${ }^{*}$, M. Sharanya ${ }^{2}$, M. Srikanth Reddy ${ }^{1}$, \\ G. Sai Ram Sandeep ${ }^{1}$, M. Abhilash ${ }^{1}$, J. Venkatesh Yadav ${ }^{3}$, P. Purushotham ${ }^{1}$, \\ I. Kesavulu Naidu ${ }^{2}$, A. Uma Chowdhary ${ }^{3}$, K. Sandhya Rani ${ }^{3}$, V. Pavani ${ }^{3}$, K. Vishwas $^{3}$, \\ B. Manoranjan Reddy ${ }^{3}$, Ch. Srinath ${ }^{4}$, P. Swapna ${ }^{4}$, A. Sreeja ${ }^{4}$, E. Kumar ${ }^{5}$, \\ Y. Narasimha Reddy ${ }^{1}$ and K. Dhana Lakshmi ${ }^{1}$ \\ ${ }^{1}$ Department of Veterinary Microbiology, ${ }^{2}$ Department of Veterinary Biotechnology, \\ ${ }^{3}$ Department of Veterinary Pathology, ${ }^{4}$ Department of Veterinary Parasitology, ${ }^{5}$ Department \\ of Veterinary Public Health and Epidemiology, College of Veterinary Science, P. V. N. R. \\ Telangana Veterinary University, Rajendranagar, Hyderabad-500030, Telangana, India
}

*Corresponding author

\section{A B S T R A C T}

Dermatophytes are a group of closely related keratinophilic fungi that can invade keratinized humans and animals tissues such as skin, hair and nails causing dermatophytosis. They are an important cause of superficial fungal infection.

\begin{tabular}{|l|}
\hline Ke y w or d s \\
Dermatophytes, \\
PCR, Chitin \\
synthase 1 gene
\end{tabular}
Conventional methods like potassium hydroxide $(\mathrm{KOH})$ direct microscopy and fungal culture lacks the ability to make an early and specific diagnosis, as it takes about four weeks for the culture to grow on Sabourauds dextrose agar. So the present study was carried out by Polymerase chain reaction (PCR) using group specific primers targeting dermatophyte specific sequence of chitin synthase 1 (CHS1) gene with an estimated product size of 288bp. Hair follicles were collected from four different horses that were exhibiting Dermatophytosis clinical lesions on skin. DNA was extracted from hair follicles by Phenol chloroform method and the extracted DNA was subjected for PCR. Studies showed that all the 4 samples were positive for the dermatophytic infection as they had an expected product size at $288 \mathrm{bp}$. Results indicate that PCR may be considered as rapid test for the diagnosis of dermatophytosis and can aid the clinician in initiating prompt and appropriate antifungal therapy. Early detection of such may further aid in the prevention of spreading and elimination of highly important zoonotic dermatophytes.

\section{Introduction}

Dermatophytes are a group of keratinophilic fungi that cause dermatophytoses which are highly contagious fungal infections of the skin that affect horses and other animals of all ages and breeds. Dermatophytes produce proteolytic enzymes, keratinases, which are able to hydrolyze keratin the main protein constituent of hair, nails and skin. The 
infections can be mild to severe, depending on the host immune response (Akcaglar et al., 2011; Emmons, 1955; Weitzmann and Summerbell, 1995). In horses, Microsporum and Trichophyton species have been reported to be the causative agents of dermatophytosis (Quinn and Markey, 2003; Ural et al., 2008).

Trichophyton equinum is the most commonly involved agent and has been reported in many countries (Hasegawa and Usui, 1975). Other Trichophyton spp. that have been isolated include Trichophyton mentagrophytes (Shimozawa et al., 1997; Quinn and Markey, 2003) and Trichophyton verrucosum (Shimozawa et al., 1997; Khosravi and Mahmoudi, 2003). Ringworm can affect horses of all ages, young animals (under two years) are predisposed to getting symptomatic infections, and in part this is due to a delay in the development of host immunity. T. equinum var. equinum and $T$. equinum var. autotrophicum have their natural host in horses. T. equinum var. equinum constitutes the major cause of equine dermatophytosis, followed by $M$. equinum and $M$. gypseum. Other dermatophytes are also reported, such as $M$. canis, $T$. mentagrophytes and $T$. verrucosum. Rarely recorded dermatophytes are $T$. quinckeanum, T. terrestre, $T$. ajelloi, $T$. bullosum, $M$. praecox and Arthroderma vanbreuseghemii (Refai et al., 2016). Because the infection is almost always follicular in horses, the most consistent clinical sign is one or many circular patches of alopecia with variable scaling and crusting. Fungal culture of affected hair and scale is the most effective way to diagnose ringworm infection, to identify the dermatophyte. The advent of molecular technology has enabled the development of techniques like polymerase chain reaction, which is a highly sensitive and specific test and can be used for diagnosis of various microorganisms including fungal pathogens. Hence in the present study we have tried to detect the presence of the dermatophytes by using gene specific chitin synthase 1 ( $\mathrm{CHS} 1)$ gene primers when we have received a case with a complaint of patchy hair loss and skin lesions on different parts of the body of the animal.

\section{Materials and Methods}

\section{Sample collection}

Plucked hair samples were collected from the margins of the lesions after cleaning and disinfecting with $70 \%$ alcohol as described by Elewski (1995). Hairs were plucked by pulling them with thumb forceps (Quinn et al., 1994). Samples collected from the different parts of the lesion are pooled together as one sample. All collected animal samples $(\mathrm{N}=4)$ were from the male animals. Collected samples were placed in sterile envelopes in separate polythene bags, and transported as dry packet (Guillot et al., 2001) to the microbiology laboratory of College of Veterinary Science, Rajendranagar.

\section{Direct microscopic examination of samples}

Small samples of hair follicle were placed on a microscope slide and 1 to 2 drops of $10 \%$ potassium hydroxide added. A cover slip was applied and the slide was gently heated over flame as described by Hainer (2003). Each treated slide was carefully examined under low objective $(\times 10)$ and high $(\times 40)$ power objectives to observe for presence of diagnostic fungal forms.

\section{Laboratory culture of dermatophytes}

Sabouraud dextrose agar (SDA) containing chloramphenicol (40mg/L), cycloheximide $(500 \mathrm{mg} / \mathrm{L})$ and nicotinic acid $(100 \mu \mathrm{g} / \mathrm{ml})$ which is a selective media was used for primary isolation of dermatophytes. The SDA plates were inoculated with the sample and incubated at room temperature for one to two weeks. 
Fig.1 Horse showing dermatophytosis clinical lesions on skin

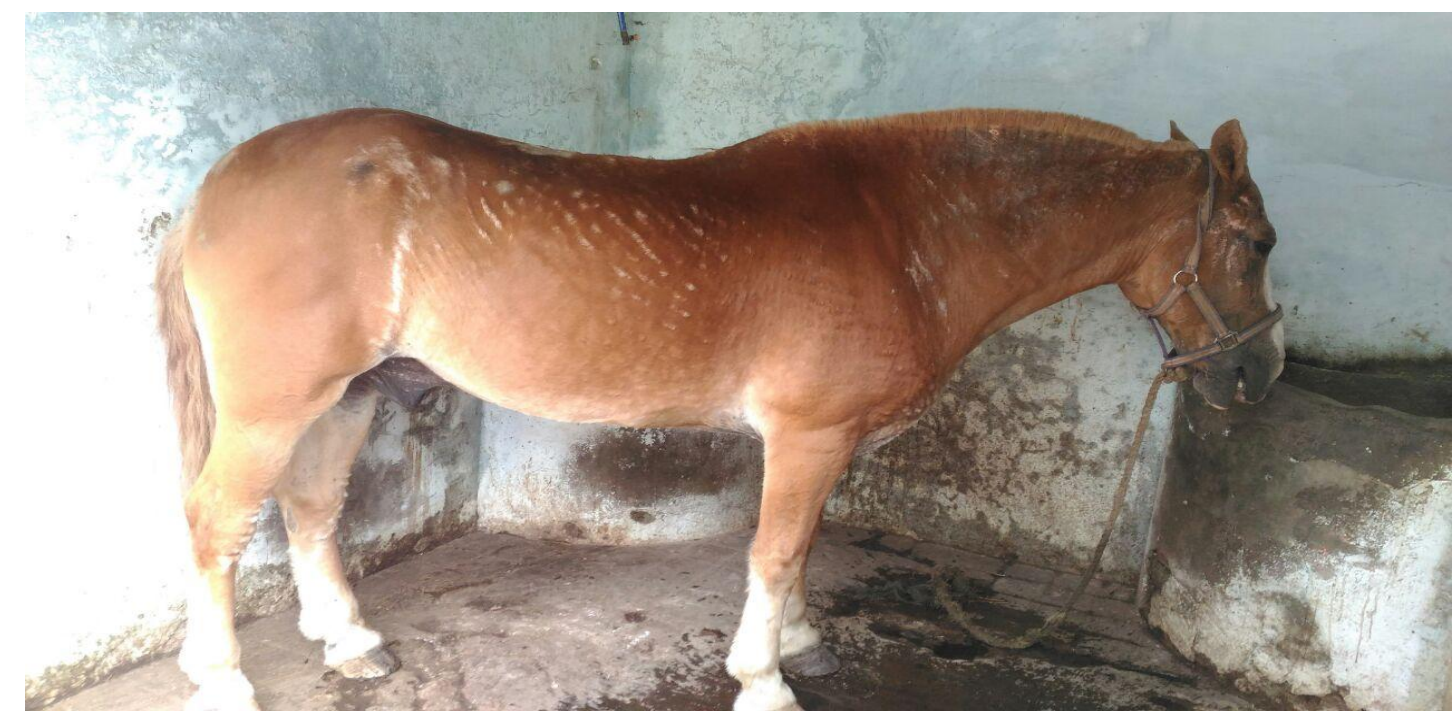

Irregular patchy skin lesions are seen on the skin surface of the animal

Fig.2 Microscopic image of microconidia (40X)

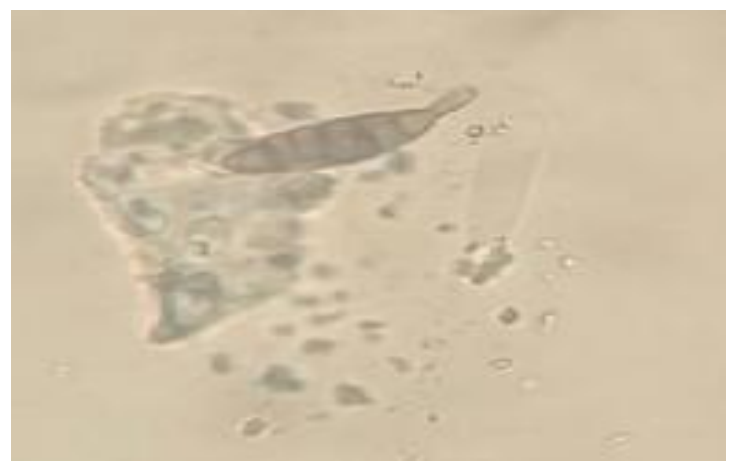

Presence of clavate to pyriform microconidia were observed under the microscope using $10 \%$ potassium hydroxide

Fig.3 Molecular detection of dermatophytes by PCR amplification of chitin synthase-1 gene

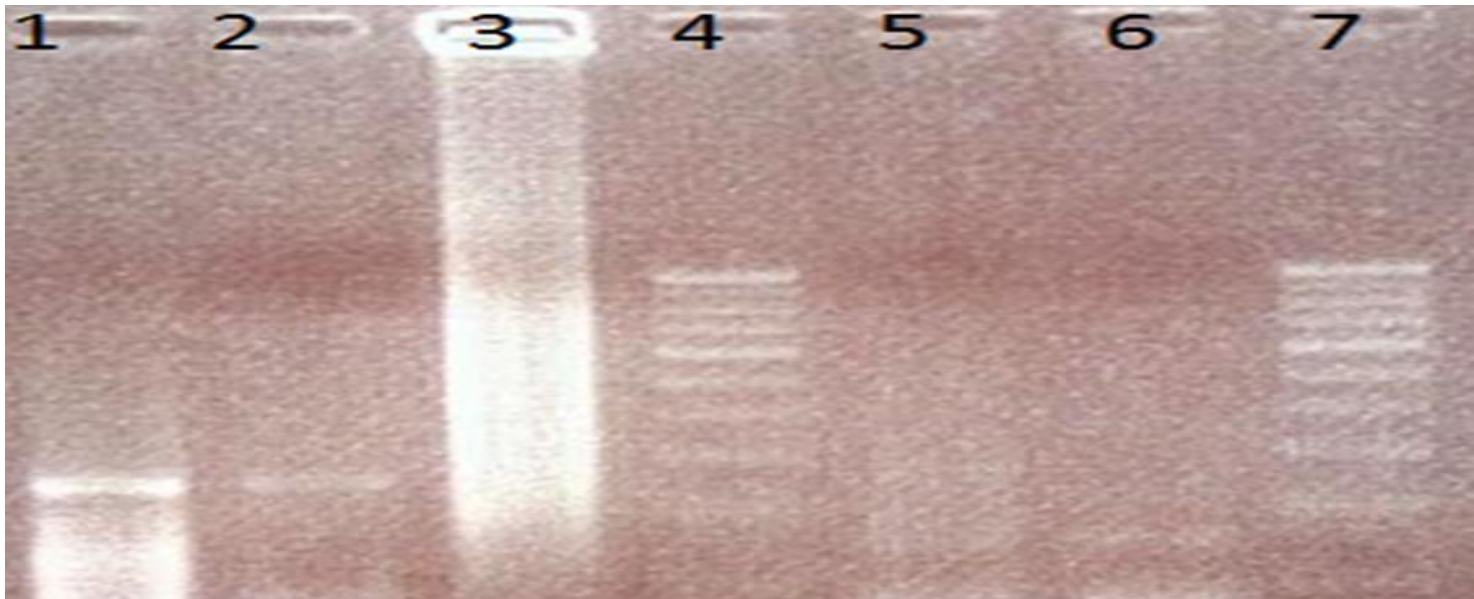

Lane 1, 2, 3, 5: samples showing an amplicon at 288bp; Lane4, 7: 100bp marker; Lane6: Negative Control 


\section{Molecular detection by PCR}

DNA extraction was performed by crushing the samples in liquid nitrogen. The crushed specimen were suspended in $200 \mu \mathrm{l}$ of TrisEDTA buffer and subjected to repeated freezing and thawing. Then, $300 \mu \mathrm{l}$ of $0.1 \%$ Triton X-100 (pH 8) and $2 \mu$ lof proteinase $\mathrm{K}$ solution $(20 \mathrm{mg} / \mathrm{ml})$ were added and incubated for $2 \mathrm{~h}$ at $65^{\circ} \mathrm{C}$.

The extracted DNA was purified by the phenol-chloroform method and resuspended in $30 \mu \mathrm{l}$ of nucleus free water. PCR was performed using group specific primers at a final concentration of $1 \mu \mathrm{M}$ targeting dermatophyte specific sequence of Chitin Synthase 1 (CHS1) gene (Jaya Garg et al., 2009) PANDER FWD(5-'GAA GAA GAT TGT CGT TTG CAT CGT CTC-3’)

PANDER REV (5-'CTC GAG GTC AAA AGC ACG CCA GAG-3'), amplifying a DNA fragment of $288 \mathrm{bp}$. The reaction mixture was initially denatured at $94^{\circ} \mathrm{C}$ for 3 min, followed by 30 cycles of denaturation at $94^{\circ} \mathrm{C}$ for $60 \mathrm{~s}$, annealing at $60^{\circ} \mathrm{C}$ for $30 \mathrm{~s}$, and extension at $72^{\circ} \mathrm{C}$ for $30 \mathrm{~s}$.

This was followed by a final extension step for $10 \mathrm{~min}$ at $72^{\circ} \mathrm{C}$ in a thermal cycler. Thereafter, bands were visualized under UV illuminator and amplicon of $288 \mathrm{bp}$ was taken as positive for dermatophytes.

\section{Results and Discussion}

The current study examined four horses exhibiting Dermatophytic lesions on anatomical sites of the body i.e., limbs, tail, head and abdominal region which were suggestive of dermatophytosis (Fig. 1). All the 4 samples were first examined under direct microscopy by $\mathrm{KOH}$ which revealed the presence of clavate to pyriform microconidia (Fig. 2).
When further preceded for culturing on SDA for 3 weeks, colonies were flat, but some developed gentle folds, white to buff in colour, suggesting Dermatophyte infection. Simultaneously, molecular detection was also carried out by isolation of DNA from the hair samples and amplification with Dermatophyte specific Chitin Synthase 1 (CHS1) gene primers.

All the 4 samples had an amplicon at expected size of 288bp (Fig. 3). Our study showed that molecular methods had a good potential to directly detect dermatophytes in clinical specimens; however detection aiming at the species level are yet to be carried out for routine clinical laboratories. Furthermore, 4 cases which were positive both by $\mathrm{KOH}$ microscopy and culture were also positive by PCR thus demonstrating that PCR was not only equally sensitive as the gold standard tests but also faster and easier to perform.

It may therefore be concluded that PCR targeting the CHS1 gene may be considered as a rapid initial screening test for detection of dermatophytes in horses suspected of dermatophytoses, which can aid the clinician in initiating a prompt and appropriate antifungal therapy.

This technique is not only rapid but also simple and cheap in comparison to other methods for detection of dermatophytes.

\section{Acknowledgements}

We are grateful to the technical staff of the Stable farm who assisted in collection of samples.

\section{References}

Akcaglar S, Ene B, Toker SC, Ediz B, Tunali $S$ and Tore O (2011). A comparative study of dermatophyte infections in 
Bursa, Turkey. Medical Mycology, 49(3): 602-607.

Elewski BE (1995). Practice - based confirmation of Onychomycosis: US nationwide prospective survey. Archives of International Medicine, 162(18): 2133-2138.

Emmons CW (1955). Mycoses of animals. Advance Veterinary Science, 2(1): 4763.

Hasegawa A and Usui K. (1975). Isolation of Trichophyton equinum and Microsporum canis from equine dermatophytosis. Journal of Medical Mycology, 16(1): 11-13.

Quinn PJ \& Markey RK (2003). Concise Review of Veterinary Microbiology.
Blackwell Publishing Oxford, United Kingdom. Pp. 74- 75.

Refai, M.K. et al., (2016). Monograph on Equine Mycoses and Mycotoxicoses A guide for postgraduate students,

Shimozawa K, Anzai T, Kamada M and Takatori K (2001). Fungal and bacterial isolation from racehorses with infectious dermatosis. Journal of Equine Science, 8(4): 89-93.

Ural K, Cingi CC and Civelek T (2008). Mycotic Blepharitis Due to Trichophyton equinum in a Horse and Treatment with Topica.

Weitzman I and Summerbell RC (1995). The Dermatophytes. Clinical Microbiology Review, 8(2): 240-269.

\section{How to cite this article:}

Kalyani Putty, J. Shiva Jyothi, M. Sharanya, M. Srikanth Reddy, G. Sai Ram Sandeep, M. Abhilash, J. Venkatesh Yadav, P. Purushotham, I. Kesavulu Naidu, A. Uma Chowdhary, K. Sandhya Rani, V. Pavani, K. Vishwas, B. Manoranjan Reddy, Ch. Srinath, P. Swapna, A. Sreeja, E. Kumar, Y. Narasimha Reddy and Dhana Lakshmi, K. 2018. PCR as a Rapid Diagnostic Tool for Detection of Dermatophytes. Int.J.Curr.Microbiol.App.Sci. 7(04): 20212025. doi: https://doi.org/10.20546/ijcmas.2018.704.232 